Check for updates

Cite this: RSC Adv., 2017, 7, 45757

Received 14th July 2017

Accepted 19th September 2017

DOI: $10.1039 / \mathrm{c} 7 \mathrm{ra0} 7724 \mathrm{f}$

rsc.li/rsc-advances

\title{
The use of dual reductants in gold nanoparticle syntheses
}

\author{
E. G. Wrigglesworth (iD)* and J. H. Johnston (1D
}

We present novel syntheses of gold nanoparticle colloids using two reducing agents simultaneously as dual reductants. These colloids were characterised by absorption spectroscopy, hydrodynamic distribution data and transmission electron microscope images, and compared to those produced by single reducing agents. The use of tannic acid and sodium borohydride together has resulted in small spherical particles and a brown coloured colloid, and has demonstrated how the size of the reducing/stabilising agent can directly influence the size of the resulting nanoparticles. Further, the simultaneous use of tannic acid and ascorbic acid has given purple/blue and blue/grey shades resulting from quasi-spherical and anisotropic particles respectively. This illustrates how dual reductants can be utilised as a means to control particle shape.

\section{Introduction}

Gold nanoparticles are well known and utilised for their bright and attractive colours. Since the 17th century they have been used to give red and purple shades to glass, ${ }^{1}$ and today their controllable colour is utilised in sensor, imaging and design applications..$^{2-6}$ The observed colour results from localised surface plasmon resonance (LSPR); the resonance oscillation of nanoparticle conduction electrons upon interaction with incoming electromagnetic radiation. ${ }^{7}$ This gives rise to intense absorptions in the visible spectrum. The energy of these absorptions, and hence the colour of the nanogold, can be controlled by modifying the size and shape of the particles. For example, spherical gold nanoparticles $20 \mathrm{~nm}$ in diameter absorb light with a wavelength of approximately $520 \mathrm{~nm}$, giving a red coloured colloid, and increasing the size of the particles results in the LSPR band shifting to longer wavelengths and becoming broader. ${ }^{8}$ Correspondingly, the colloidal colour changes through purple to grey for very large particles and aggregates. Further, nanorods possess two size dimensions and so give rise to two LSPR absorptions. When synthesised by a seeding growth approach using cetyltrimethylammonium bromide (CTAB), nanorods with aspect (longitudinal to transverse) ratios of 13 and 18 give blue coloured colloids. ${ }^{9}$ However CTAB is known to be toxic and so new environmentally safe methods to extend the colour range are desirable. ${ }^{10}$

Gold nanoparticles are commonly synthesised by the chemical reduction method in which $\mathrm{Au}^{3+}$ ions are reduced by a suitable agent. Common reducing agents include trisodium citrate, ${ }^{11-13}$ tannic acid, ${ }^{14,15}$ sodium borohydride, ${ }^{16-18}$ ascorbic

School of Chemical and Physical Sciences, Victoria University of Wellington, PO Box 600, Wellington 6140, New Zealand. E-mail: emma.wrigglesworth@vuw.ac.nz acid, ${ }^{19-21}$ and amine molecules. ${ }^{22,23}$ The addition of a stabilising agent such as polyvinylpyrrolidone (PVP) is often required..$^{24,25}$

Here we present two novel nanogold syntheses in which two reducing agents are used simultaneously as dual reductants. To the best of our knowledge these syntheses have not been reported in the literature before now. We demonstrate that when tannic acid and sodium borohydride are used together, a brown coloured colloid results from small spherical particles. The use of tannic acid and ascorbic acid together gives purple/blue and blue/grey shades resulting from quasi-spherical and anisotropic particles respectively. No structure directing agents such as CTAB are used. These reproducible syntheses, each consisting of two simple steps, were carried out in aqueous media at room temperature. They provide advantages over other common methods utilised to obtain gold nanoparticles of these types.

Previously reported two reductant syntheses have utilised a block copolymer which is a very mild reducing agent and acts mostly as a stabilising agent to control particle size. ${ }^{26}$ The addition of trisodium citrate as a second reducing agent in this report has increased the concentration of spherical particles. Anisotropic particles were not formed. Our synthesis of brown nanoparticles has extended this approach by using the size of the stabilising/reducing agent to directly control the size of the nanoparticles. However our synthesis of blue and grey nanoparticles is completely distinct from this in that we utilise two highly effective reducing agents to significantly alter the mechanism of nanoparticle formation from when either reducing agent is used alone, resulting in different sized and shaped particles including anisotropic particles. To the best of our knowledge, we report for the first time the use of small molecule dual reductants with very similar reducing properties, added in very quick succession, to control nanoparticle shape and produce anisotropic particles. This is achieved without the 
use of a toxic growth directing agent such as CTAB, instead utilising the non-toxic, biodegradable and biocompatible chemicals tannic acid and ascorbic acid.

\section{Experimental}

\section{Brown nanogold synthesis}

Sample A was synthesised by adding $\mathrm{HAuCl}_{4}(20 \mu \mathrm{L}, 3.36 \mathrm{wt} \%$ $\mathrm{Au})$ to distilled water $(30 \mathrm{~mL})$ in a glass beaker, while stirring at room temperature. Tannic acid $(200 \mu \mathrm{L}, 1 \mathrm{wt} \%)$ was then added. Sodium borohydride $\left(1200 \mu \mathrm{L}, 0.05 \mathrm{~mol} \mathrm{dm}^{-3}\right)$ was added $30 \mathrm{~s}$ later (before any colour change resulting from $\mathrm{Au}^{3+}$ reduction by tannic acid). A colour change was observed within $10 \mathrm{~s}$ of the addition of sodium borohydride and the solution was allowed to stir for a further $45 \mathrm{~min}$. The final $\mathrm{pH}$ of the sample was 8.0. The colour of the colloid was observed to change overnight, and to continue to change slightly over the next five to six days. Therefore characterisations were carried out seven days after synthesis. The colloid was stable for at least six months.

To act as a comparison, gold colloids made using only sodium borohydride (sample 1) and only tannic acid (sample 2) were also synthesised. $\mathrm{HAuCl}_{4}(20 \mu \mathrm{L}, 3.36 \mathrm{wt} \% \mathrm{Au})$ was added to distilled water $(30 \mathrm{~mL})$ in a glass beaker, while stirring at room temperature. Sodium borohydride (sample 1, $1200 \mu \mathrm{L}$, $0.05 \mathrm{~mol} \mathrm{dm}^{-3}$ ) or tannic acid (sample 2, $200 \mu \mathrm{L}, 1 \mathrm{wt} \%$ ) was added and the solution allowed to stir for a further $45 \mathrm{~min}$.

For further comparison, a colloid made using sodium borohydride and polyvinylpyrrolidone (PVP) as the stabilising surfactant was synthesised (sample 3). $\mathrm{HAuCl}_{4}(20 \mu \mathrm{L}, 3.36 \mathrm{wt} \%$ $\mathrm{Au})$ was added to distilled water $(30 \mathrm{~mL})$ in a glass beaker, while stirring at room temperature. PVP $(100 \mu \mathrm{L}, 2 \mathrm{wt} \%)$ was added followed by sodium borohydride $\left(1200 \mu \mathrm{L}, 0.05 \mathrm{~mol} \mathrm{dm}^{-3}\right)$. The solution was allowed to stir for a further $45 \mathrm{~min}$.

\section{Blue nanogold synthesis}

$\mathrm{HAuCl}_{4}(10 \mu \mathrm{L}, 3.36 \mathrm{wt} \% \mathrm{Au})$ was added to distilled water $(10 \mathrm{~mL})$ in a glass vial, while stirring at room temperature. Subsequently a volume of tannic acid $(1 \mathrm{wt} \%)$ was added $(50 \mu \mathrm{L}$, $100 \mu \mathrm{L}$ and $500 \mu \mathrm{L}$ for samples B, C and D respectively). Ascorbic acid $(50 \mu \mathrm{L}, 5 \mathrm{wt} \%)$ was added $30 \mathrm{~s}$ later (before any colour change resulting from $\mathrm{Au}^{3+}$ reduction by tannic acid). A colour change was observed within $10 \mathrm{~s}$ of the addition of ascorbic acid and the solution was allowed to stir for a further $15 \mathrm{~min}$. The final $\mathrm{pH}$ of all the samples was 2.4. All characterisations were performed on the day of synthesis.

To act as a comparison, gold colloids made using only tannic acid (sample 4) and only ascorbic acid (sample 5) were also synthesised. $\mathrm{HAuCl}_{4}(10 \mu \mathrm{L}, 3.36 \mathrm{wt} \% \mathrm{Au})$ was added to distilled water $(10 \mathrm{~mL})$ in a glass vial, while stirring at room temperature. Tannic acid (sample 4, $100 \mu \mathrm{L}, 1 \mathrm{wt} \%$ ) or ascorbic acid (sample $5,50 \mu \mathrm{L}, 5 \mathrm{wt} \%$ ) was added and the solution allowed to stir for a further $45 \mathrm{~min}$.

\section{Characterisation}

Absorption spectra of gold colloids were recorded with an Agilent 8453 spectrophotometer using disposable plastic cuvettes with a path length of $1 \mathrm{~cm}$. Backgrounds were run using distilled water. Transmission electron microscopy (TEM) images were obtained using a JEOL 2010 electron microscope. Colloid samples were drop-cast onto 200 mesh copper grids and allowed to air dry. A JEOL EC-52000IC ion cleaner was used to plasma treat each sample for $15 \mathrm{~min}$ at $\sim 300 \mathrm{~V}$ to remove organic substances. Particle diameters were determined using the software ImageJ. Hydrodynamic radius measurements were obtained using a Zetasizer Nano ZS instrument, using disposable plastic cuvettes with a path length of $1 \mathrm{~cm}$. A $4 \mathrm{~mW} \mathrm{He-Ne}$ laser was employed at a wavelength of $633 \mathrm{~nm}$ and a backscattered light detection angle of $173^{\circ}$.

\section{Results and discussion}

The syntheses presented here have utilised two reducing agents simultaneously to produce gold nanoparticles with interesting sizes and/or shapes. First, the use of tannic acid and sodium borohydride simultaneously has resulted in a colloid with optical properties that differed from when either reducing agent was used alone (Fig. 1(a) and (b)). When sodium borohydride was used alone to reduce $\mathrm{Au}^{3+}$ (sample 1), a pale pink solution resulted with an LSPR peak centred at $519 \mathrm{~nm}$ in the absorption spectrum. This compares well to literature results where $5.5 \pm$ $0.2 \mathrm{~nm}$ spherical particles resulted in a red/pink solution with a $\lambda_{\max }$ value of $515 \mathrm{~nm} .{ }^{18}$ The use of tannic acid alone in sample 2 gave a red coloured solution and a LSPR band at $528 \mathrm{~nm}$. This is consistent with literature reports in which similar LSPR bands have resulted from spherical particles with average diameters ranging from $8 \mathrm{~nm}$ to $25 \mathrm{~nm} .{ }^{14,15}$ The reducing agent concentrations in these samples were chosen such that complete reduction of $\mathrm{Au}^{3+}$ ions was achieved.

The use of these two reducing agents together gave a brown coloured colloid (sample A). The absorption spectrum of sample A has shown a strong absorption in the ultraviolet region that decays into the visible region, with superimposed LSPR bands at 430 and $490 \mathrm{~nm}$ (Fig. 1(a)). This is consistent with the colour observed and is indicative of the presence of very small gold nanoparticles. ${ }^{27}$ The two peaks suggest two dominating particle sizes but because they were not well defined there are likely a significant number of other nanoparticle sizes also present around these size ranges. The hydrodynamic diameter distribution data has shown two peaks centred at $2.6 \mathrm{~nm}$ and $7.0 \mathrm{~nm}$ and so supports the presence of two main particle sizes (Fig. 1(c)). Because larger particles absorb light of a lower energy, ${ }^{8}$ this suggests that $\sim 2.6 \mathrm{~nm}$ particles were responsible for the absorption band at $430 \mathrm{~nm}$, and $\sim 7.0 \mathrm{~nm}$ particles for that at $490 \mathrm{~nm}$, collectively resulting in the brown colour.

TEM analysis of sample A has revealed a large number of small, spherical nanoparticles as predicted from the absorption spectrum (Fig. 1(d) and (e)). The majority of the particles had diameters between $1 \mathrm{~nm}$ and $8 \mathrm{~nm}$, however some larger particles were also observed (Fig. 1(f)). Although care was taken to ensure the TEM images obtained were representative of the entire sample, the size analysis did not confirm the two main sizes of nanoparticles that the absorption and hydrodynamic diameter data have suggested. 
(a)
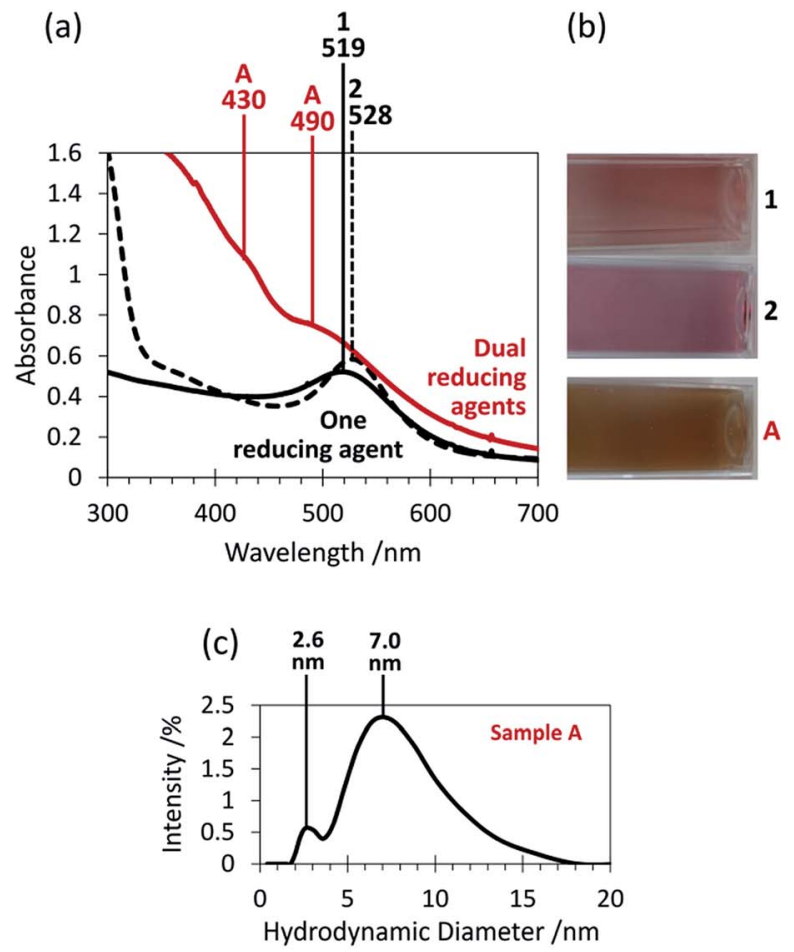

(d)

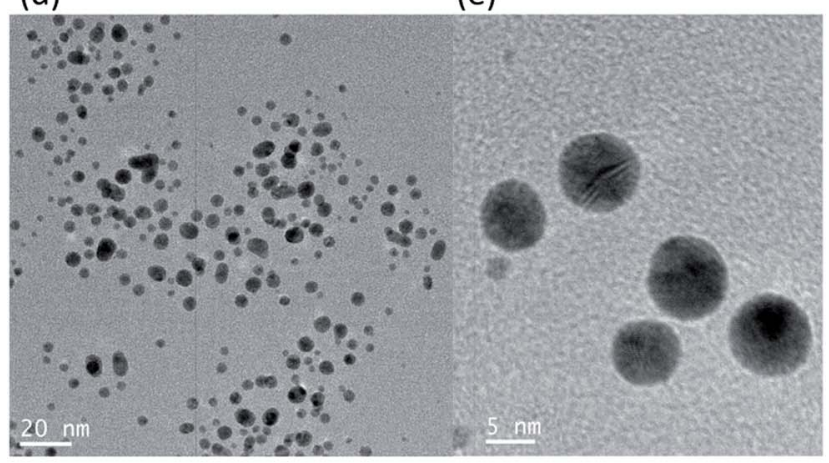

(f)

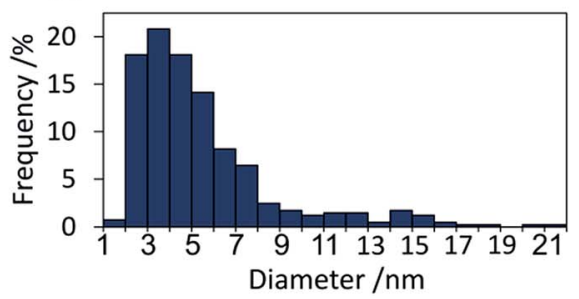

Fig. 1 (a) Absorption spectra of gold colloid sample A (dual reductants tannic acid and sodium borohydride) as compared to gold colloid samples 1 and 2 (single reductants sodium borohydride and tannic acid respectively), (b) corresponding photographs of the colloids, (c) hydrodynamic diameter intensity distribution for sample A, (d) typical low magnification TEM image of sample $A$, (e) typical high magnification TEM image of sample A, (f) histogram of sample A nanoparticle diameters, based on 403 particles imaged via TEM, calculated using ImageJ.

Tannic acid is an effective reducing agent and is capable of reducing $\mathrm{Au}^{3+}$ at room temperature and forming a high concentration of nanoparticles, however in this case it is its role as a stabilising agent that is of more importance. When tannic acid was replaced in the synthesis by PVP (sample 3), the brown colour of the resulting colloid was visually similar to that of sample A, as reflected by the fact that both colloids gave a band in the absorption spectra at $490 \mathrm{~nm}$ (Fig. 2). Because PVP cannot act as a reductant at room temperature but only as a stabiliser, the similarity between the colloids suggests that tannic acid played a mostly stabilising role in the formation of the sample A colloid. However the absorption spectrum for sample 3 did not possess the peak at $430 \mathrm{~nm}$ that the sample A colloid did and therefore the presence of the nanoparticles that have resulted in this peak ( $\sim 2.6 \mathrm{~nm}$ in diameter) must be due to the addition of tannic acid. It could be that these $\sim 2.6 \mathrm{~nm}$ nanoparticles have formed as a result of a favourable nanoparticle-tannic acid ratio. These nanoparticles are on the same size scale as the stabilising tannic acid molecules $(\sim 2.5 \mathrm{~nm}$ from one side of a tannic acid molecule to the other). This suggests there might be a favourable number of tannic acid molecules per nanoparticle, and if it is beneficial to maximise nanoparticle coverage to maximise stabilisation then this would drive the formation of nanoparticles of a certain size. If a tannic acid molecule is approximated as a circle with an area of $\sim 4.9 \mathrm{~nm}^{2}$ then it would require four molecules to completely coat a nanoparticle with a diameter of $2.5 \mathrm{~nm}$ (surface area $=$ $19.6 \mathrm{~nm}^{2}$ ). If this is the tannic acid to gold ratio that is most favoured then this would drive the formation of $2.5 \mathrm{~nm}$ diameter gold nanoparticles, explaining the presence of the peak at $2.6 \mathrm{~nm}$ in the hydrodynamic diameter distribution. Nanoparticles of this size fall into the range known to be most effective for catalysis (typically less than $5 \mathrm{~nm}$ ). ${ }^{28-31}$ This concept of using the size of the tannic acid stabilising agent to directly control the size of the resulting particle is a novel approach and has significant potential in catalysis applications. Therefore further research into optimising this process would be beneficial for this field of research.

The absorbance intensity in the spectrum of sample 3 was much lower and the colloid much paler than for sample A, even though the gold concentration was identical and the sodium borohydride added in excess. It is likely that a large number of

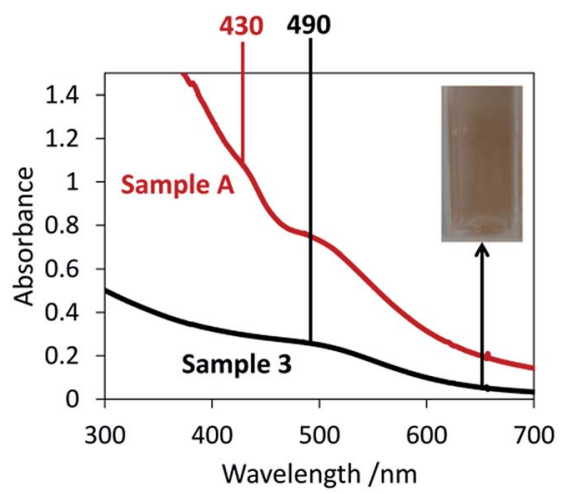

Fig. 2 Absorption spectra of gold colloid sample 3 (reductant sodium borohydride and stabiliser PVP) as compared to gold colloid sample A (dual reductants tannic acid and sodium borohydride), inset: photograph of gold colloid sample 3 . 
the nanoparticles in sample 3 were too small to undergo LSPR and so did not contribute to the colour and were not observed via absorption spectroscopy. The synthesis of sample A is therefore advantageous in applications where a strong brown colour is desirable, for example in certain design applications.

This dual reductant synthesis of nanogold has similarities with that given in the literature, in that one of the reducing agents plays a significant role in particle stabilisation. ${ }^{26}$ However where the second reductant simply increases the concentration of nanoparticles in the reported synthesis, the use of tannic acid and sodium borohydride together here has resulted in an overall decrease in the nanoparticle size. This control over size is an important advancement. Further, the choice of reducing agents in dual reductant syntheses can completely change the mechanism of nanoparticle formation, as shown below.

Tannic acid and ascorbic acid are both effective reducing agents, and are both able to reduce $\mathrm{Au}^{3+}$ at room temperature within comparable timeframes. Their use as dual reductants has resulted in particles with optical properties that differ from when tannic acid or ascorbic acid was used alone (Fig. 3(a)). Like in sample 2, the use of tannic acid alone in sample 4 gave a red coloured solution and an LSPR band at $528 \mathrm{~nm}$. When ascorbic acid was used alone (sample 5), this produced a purple colloid with a slightly wider LSPR band at $553 \mathrm{~nm}$. This colloid was analysed via TEM in this study and found to contain spherical and quasi-spherical particles with an average diameter of $40 \pm 14 \mathrm{~nm}$, consistent with the nanospheres $30-40 \mathrm{~nm}$ in diameter and red/purple colloids reported in the literature. ${ }^{\mathbf{1 9}}$ Again, the reducing agent concentrations utilised have ensured complete reduction of the $\mathrm{Au}^{3+}$ ions.

The colloid changed significantly in colour to a purple/blue shade upon use of the two reducing agents together (sample B) (Fig. 3(b)). Tannic acid and ascorbic acid are both biodegradable, biocompatible and possess low toxicity. Therefore this synthesis of blue nanogold offers a significant advantage over the typical CTAB synthesis of nanorods utilised to obtain blue colours, as CTAB is known to be toxic. ${ }^{\mathbf{1 0}}$ The peak in the

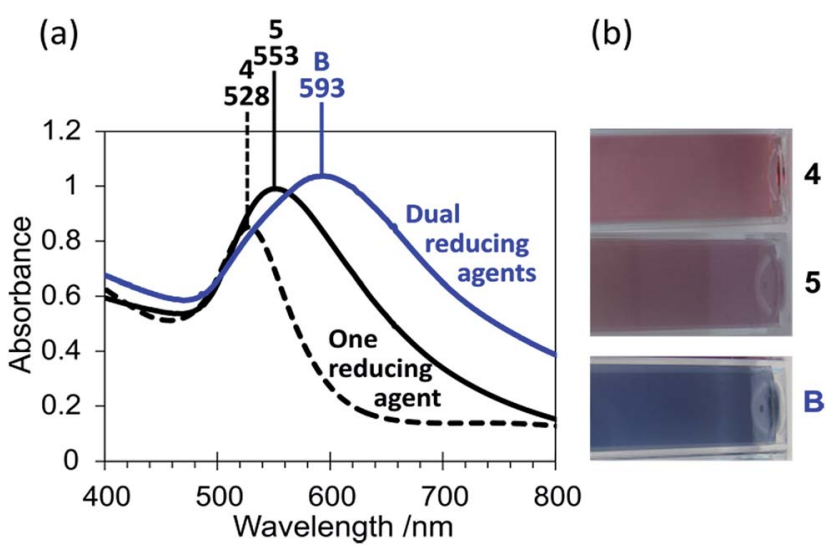

Fig. 3 (a) Absorption spectra of gold colloid sample B (dual reductants tannic acid and ascorbic acid) as compared to gold colloid samples 4 and 5 (single reductants tannic acid and ascorbic acid respectively), (b) corresponding photographs of the colloids. (a)

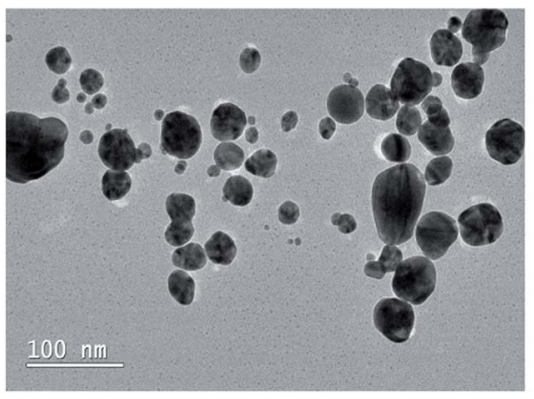

(b)

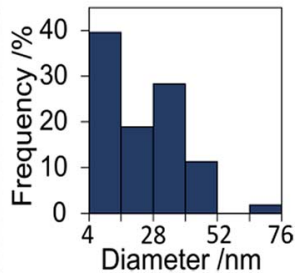

Fig. 4 (a) Typical TEM image of gold colloid sample B (dual reductants tannic acid and ascorbic acid), (b) histogram of nanoparticle diameters corresponding to image (a), calculated using ImageJ.

absorption spectrum of sample B has red-shifted from those of samples 4 and 5 to $593 \mathrm{~nm}$, which suggests a larger particle size or that some agglomeration/aggregation has occurred. Additionally the LSPR peak was much broader for sample B, consistent with the blue shade observed and indicating a greater particle size distribution or anisotropy. The TEM images of the purple/blue colloid (sample B) showed particles that were spherical or quasi-spherical in shape (Fig. 4(a)), similar in shape to those observed in TEM images of sample 5. In the absence of any observable anisotropy, the blue colour of sample B is attributed to the large range of nanoparticle sizes observed (Fig. 4(b)). A mechanism of nanoparticle growth has been developed for sample B. Although tannic acid can fully reduce $\mathrm{Au}^{3+}$, it is not quite as effective as ascorbic acid. Hence, when the tannic acid was added to the $\mathrm{Au}^{3+}$ solution initially it was not able to produce any significant quantity of nanoparticles in the short time before ascorbic acid was added (30 seconds), as indicated by the lack of any colour development. Because ascorbic acid is the stronger reducing agent, the following addition of ascorbic acid to the tannic acid and $\mathrm{Au}^{3+}$ solution resulted in nanoparticles quickly beginning to form. These partially formed nanoparticles possess layers of $\mathrm{Au}^{+}$on their surface, and $\mathrm{Au}^{+}$is easier to reduce than $\mathrm{Au}^{3+}\left(E^{\circ}\right.$ values of $+1.692 \mathrm{~V}$ and $+1.498 \mathrm{~V}$ for $\mathrm{Au}^{+}$and $\mathrm{Au}^{3+}$ respectively)..$^{32}$ Therefore tannic acid was able to act to grow the nanoparticles partially formed by ascorbic acid by capturing the early formed tannic acid nanoparticles on the surface as well as forming further nanoparticles in its own right. The large range of nanoparticle sizes observed by TEM can be attributed to this mechanism due partly to the slightly different rates of reaction between tannic acid and ascorbic acid, and that individual nanoparticles will have come into contact with different quantities of tannic acid molecules, at different stages in their growth, during this fast synthesis.

Unlike for sample A, the optical properties of the colloid could be further altered by increasing the concentration of the second reducing agent tannic acid, as shown in Fig. 5. This has resulted from the fact that the tannic acid and ascorbic acid both have reducing roles in this synthesis. The colour change from purple/blue to a blue/grey shade is consistent with the absorption spectra obtained in which the LSPR peaks for 
(a)

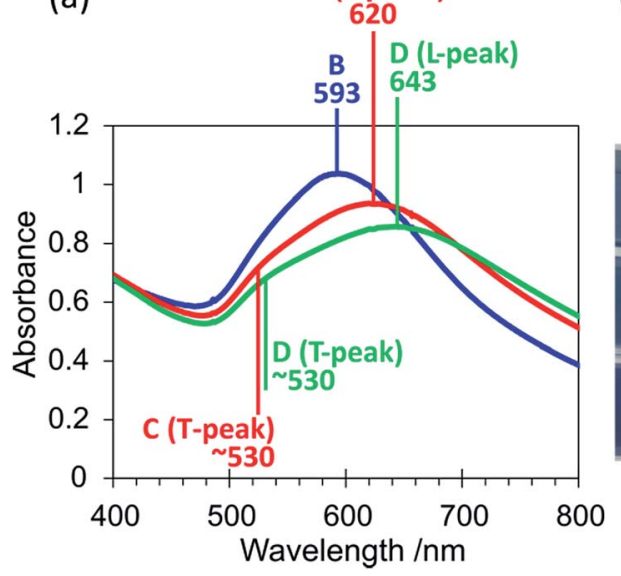

Fig. 5 (a) Absorption spectra of gold colloid samples B, C and D (dual reductants tannic acid and ascorbic acid), showing the effect of increasing tannic acid concentration (sample $B \rightarrow D$ ) $(\mathrm{L}$-peak $=$ longitudinal peak and $\mathrm{T}$-peak = transverse peak), (b) corresponding photographs of the colloids.

samples $\mathrm{C}$ and $\mathrm{D}$ were very broad and further red-shifted compared to sample B $\left(\lambda_{\max }=620 \mathrm{~nm}\right.$ and $643 \mathrm{~nm}$ for samples $\mathrm{C}$ and $\mathrm{D}$ respectively). This suggests that agglomeration or aggregation has occurred and/or that there were anisotropic particles present in these colloids. The absorption spectra of samples $\mathrm{C}$ and $\mathrm{D}$ have additionally shown a shoulder at $\sim 530 \mathrm{~nm}$ which is reminiscent of the transverse LSPR band commonly seen when nanorods are present. This peak was poorly separated from the main, longitudinal band (at $620 \mathrm{~nm}$ and $643 \mathrm{~nm}$ ) suggesting the presence of polydisperse anisotropic particles or aggregates/agglomerates. TEM analysis of sample D has confirmed that significant anisotropy was present (Fig. 6). The structures observed by TEM may be split into two categories; (i) smaller branched structures with diameters in the range of $20-40 \mathrm{~nm}$ (Fig. 6(a)), and, (ii) larger particles that were overall essentially spherical but had an uneven surface of nanoscale protrusions (Fig. 6(b)). The larger spherical particles of category (ii) had an average 'outer' diameter (including

(a)

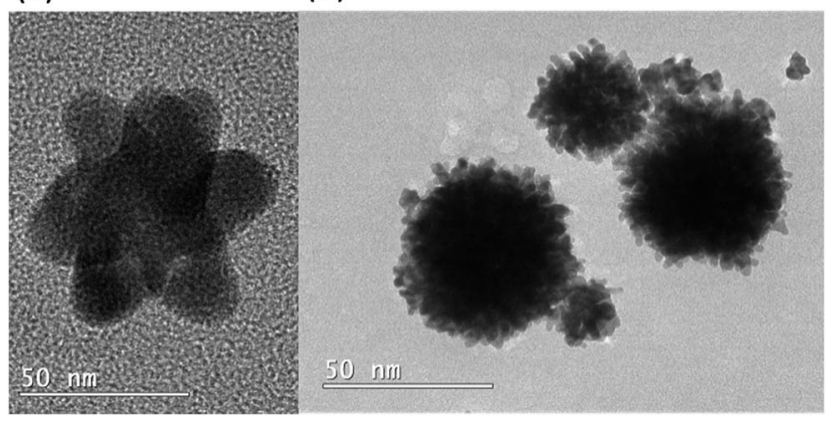

Fig. 6 Typical TEM images of gold colloid sample D (dual reductants tannic acid and ascorbic acid), showing the two particle types observed: (a) small branched structures, and, (b) larger spherical particles with nanoprotrusions. protrusions) of $56 \pm 4 \mathrm{~nm}$ as compared to an average 'inner' diameter (excluding protrusions) of $40 \pm 2 \mathrm{~nm}$ (calculated in ImageJ). The nanoprotrusions on these particles have induced multiple LSPR modes that were similar to the longitudinal mode of gold nanorods, and have resulted in a broad redshifted LSPR band and consequently a blue/grey coloured colloid. The poor resolution between the longitudinal and transverse peaks in the absorption spectra can be explained by a number of factors; (i) a large range of different nanoprotrusion aspect ratios, with a higher aspect ratio giving a redshifted LSPR mode, (ii) a large range of outer-to-inner ratios for the spherical nanoparticles with nanoprotrusions, and, (iii) a large range of structure sizes, from the small branched structures to the larger spherical particles.

The different densities observed in the TEM images of the smaller branched structures suggest that they consisted of smaller nanoparticles that have grown together to form anisotropic clusters. This is consistent with the spherical particles observed for sample B; it is likely that such particles have grown together by the action of excess tannic acid reducing agent to form structures such as those observed in Fig. 6. Because the overall size of many of these formations are smaller than a number of the spherical particles observed for sample B, this suggests that the particles have grown together while they were still in the process of formation, i.e. before they had grown to their full size.

In this synthesis, if the time of the ascorbic acid addition was delayed until after the colour change resulting from tannic acid reduction was observed (starting at $\sim 60$ seconds), then this changed the optical properties of the colloid. It resulted in a narrower, blue-shifted LSPR band and purple/pink colloids, resulting from a mixture of spherical particles made by tannic acid and spherical particles made by ascorbic acid (the greater the time delay, the greater the similarity to the optical properties of sample 4 tannic acid nanoparticles). This highlights that the tannic acid and the ascorbic acid need to be acting as reducing agents essentially simultaneously to achieve particle shape control and anisotropy. This is a novel demonstration of the use of two small molecule reducing agents of similar strength to control particle shape that we believe has not before been examined in the literature.

\section{Conclusions}

When two reducing agents with similar reductive capabilities are used simultaneously to produce gold nanoparticles, a simple mixture of two nanoparticle types is not produced. Instead the mechanism of nanoparticle formation has been altered and nanoparticles of different shapes and sizes result. The two syntheses developed proceed at room temperature, are carried out in aqueous media, and are simple and reproducible. The use of tannic acid and sodium borohydride is useful when small spherical nanoparticles and a strong brown colour are desirable. The use of tannic acid and ascorbic acid is environmentally safe and provides simple alternative means to produce anisotropic nanoparticles and purple/blue and blue/grey shades of colloid. 


\section{Conflicts of interest}

There are no conflicts to declare.

\section{Acknowledgements}

This research was supported by a Victoria University of Wellington Doctoral Scholarship.

\section{References}

1 F. E. Wagner, S. Haslbeck, L. Stievano, S. Calogero, Q. A. Pankhurst and K. P. Martinek, Nature, 2000, 407, 691-692.

2 X. Huang and M. A. El-Sayed, J. Adv. Res., 2010, 1, 13-28. 3 Y. Li, H. J. Schluesener and S. Xu, Gold Bull., 2010, 43, 29-41. 4 A. R. Zarei and F. Barghak, J. Anal. Chem., 2017, 72, 430-436. 5 J. H. Johnston and K. A. Lucas, Gold Bull., 2011, 44, 85-89.

6 A. Iwakoshi, T. Nanke and T. Kobayashi, Gold Bull., 2005, 38, 107-112.

7 K. L. Kelly, E. Coronado, L. L. Zhao and G. C. Schatz, J. Phys. Chem. B, 2003, 107, 668-677.

8 J. Turkevich, G. Garton and P. C. Stevenson, J. Colloid Sci., 1954, 9, 26-35.

9 N. R. Jana, L. Gearheart and C. J. Murphy, J. Phys. Chem. B, 2001, 105, 4065-4067.

10 S. Wang, W. Lu, O. Toymachenko, U. S. Rai, H. Yu and P. C. Ray, Chem. Phys. Lett., 2008, 463, 145-149.

11 J. Turkevich, P. C. Stevenson and J. Hillier, Discuss. Faraday Soc., 1951, 11, 55-75.

12 G. Frens, Colloid Polym. Sci., 1972, 250, 736-741.

13 F. Schulz, T. Homolka, N. G. Bastús, V. Puntes, H. Weller and T. Vossmeyer, Langmuir, 2014, 30, 10779-10784.

14 S. K. Sivaraman, S. Kumar and V. Santhanam, Gold Bull., 2010, 43, 275-286.

15 S. A. Aromal and D. Philip, Phys. E, 2012, 44, 1692-1696.

16 M. Brust, M. Walker, D. Bethell, D. J. Schiffrin and R. Whyman, J. Chem. Soc., Chem. Commun., 1994, 7, 801-802.
17 M. N. Martin, J. I. Basham, P. Chando and S. K. Eah, Langmuir, 2010, 26, 7410-7417.

18 C. Deraedt, L. Salmon, S. Gatard, R. Ciganda, R. Hernandez, J. Ruiza and D. Astruc, Chem. Commun., 2014, 50, 1419414196.

19 H. Tyagi, A. Kushwaha, A. Kumar and M. Aslam, Int. J. Nanosci., 2011, 10, 857-860.

20 Z. Khan, T. Singh, J. I. Hussain and A. A. Hashmi, Colloids Surf., B, 2013, 104, 11-17.

21 K. Sun, J. Qiu, J. Liu and Y. Miao, J. Mater. Sci., 2009, 44, 754758.

22 J. D. S. Newman and G. J. Blanchard, Langmuir, 2006, 22, 5882-5887.

23 M. J. Richardson, J. H. Johnston and T. Borrmann, Eur. J. Inorg. Chem., 2006, 13, 2618-2623.

24 Y. Chen, X. Gu, C. G. Nie, Z. Y. Jiang, Z. X. Xie and C. J. Lin, Chem. Commun., 2005, 33, 4181-4183.

25 M. M. Chili, V. R. Pullabhotla and N. Revaprasadu, Mater. Lett., 2011, 65, 2844-2847.

26 D. Ray, V. K. Aswal and J. Kohlbrecher, Langmuir, 2011, 27, 4048-4056.

27 M. J. Hostetler, J. E. Wingate, C. J. Zhong, J. E. Harris, R. W. Vachet, M. R. Clark, J. D. Londono, S. J. Green, J. J. Stokes, G. D. Wignall, G. L. Glish, M. D. Porter, N. D. Evans and R. W. Murray, Langmuir, 1998, 14, 17-30.

28 M. Haruta, Catal. Today, 1997, 36, 153-166.

29 G. C. Bond and D. T. Thompson, Gold Bull., 2000, 33, 41-50. 30 N. Lopez, T. V. W. Janssens, B. S. Clausen, Y. Xu, M. Mavrikakis, T. Bligaard and J. K. Nørskov, J. Catal., 2004, 223, 232-235.

31 I. Laoufi, M. C. Saint-Lager, R. Lazzari, J. Jupille, O. Robach, S. Garaedée, G. Cabailh, P. Dolle, H. Cruguel and A. Bailly, J. Phys. Chem. C, 2011, 115, 4673-4679.

32 P. Vanýsek, in CRC Handbook of Chemistry and Physics, ed. D. R. Lide, CRC Press, Boca Raton, 87th edn, 2006, pp. 8/20-8/ 29. 